\title{
AN ACTIVE FAULT-TOLERANT CONTROL FRAMEWORK AGAINST ACTUATOR STUCK FAILURES UNDER INPUT SATURATIONS
}

\author{
XIN QI ${ }^{a}$, DIDIER THEILLIOL ${ }^{b}$, YUQING HE $^{a, *}$, JIANDA HAN $^{a}$ \\ ${ }^{a}$ State Key Laboratory of Robotics, Shenyang Institute of Automation \\ Chinese Academy of Sciences, 110016, Shenyang, PR China \\ e-mail: \{qixin, heyuqing, jdhan\}@sia.cn \\ ${ }^{b}$ CRAN UMR 7039, CNRS \\ University of Lorraine, BP 70239, 54506 Vandoeuvre-les-Nancy, France \\ e-mail: didier.theilliol@cran.uhp-nancy.fr
}

\begin{abstract}
In this paper, a control framework including active fault-tolerant control (FTC) and reference redesign is developed subject to actuator stuck failures under input saturations. FTC synthesis and reference redesign approaches are proposed to guarantee post-fault system safety and reference reachability. Then, these features are analyzed under both actuator stuck failures and constraints before fault-tolerant controller switches. As the main contribution, actuator stuck failures and constraints are unified so that they can be easily considered simultaneously. By means of transforming stuck failures into actuator constraints, the post-fault system can be regarded as an equivalent system with only asymmetrical actuator constraints. Thus, methods against actuator saturations can be used to guarantee regional stability and produce the stability region. Based on this region, stuck compensation is analyzed. Specifically, an unstable open-loop system is considered, which is more challenging. Furthermore, the method is extended to a set-point tracking problem where the reachability of the original reference can be evaluated. Then, a new optimal reference will be computed for the post-fault system if the original one is unreachable. Finally, simulation examples are shown to illustrate the theoretical results.
\end{abstract}

Keywords: fault-tolerant control (FTC), actuator stuck failure, actuator constraints, reference redesign, linear matrix inequality (LMI).

\section{Introduction}

Fault-tolerant control (FTC), aiming to guarantee closed-loop system stability and maintain admissible degradation performance in the presence of component malfunctions (Noura et al., 2009), has attracted considerable attention over the last few decades (Zhang and Jiang, 2008). Taking an actuator, for example, malfunctions can be divided into two categories: faults and failures. An actuator fault signifies that partial effectiveness is lost, while an actuator failure means that total effectiveness is lost and cannot respond to the control signal. In this paper, a kind of actuator failure, namely, a stuck failure, is brought into focus, which signifies that the actuator locks at a fixed place. The actuator stuck failure has two characteristics different from actuator faults: (i) the decrease of available control input channels,

\footnotetext{
*Corresponding author
}

and (ii) constant control input to a post-fault system produced by the stuck actuator.

Compared with a number of research works on actuator faults, such as those by $\mathrm{Xu}$ et al. (2017) or Cen et al. (2015), less research focused on actuator stuck failures because the use of hardware redundancy is a traditional way to improve system reliability. In the presence of hardware redundancy, stuck actuators can be directly cut off from plants. However, this strategy is invalid in the absence of hardware redundancy, like with unmanned aerial vehicles, which have no or less hardware redundancy because of weight and volume limitations (Qi et al., 2014). Thus, the influence produced by stuck actuators has to be considered. Famularo et al. (2015) examined multiple-stuck-position actuator failures. All admissible stuck failures were covered by a set of piecewise affine systems, and then controllable sets were able to be computed according to these systems. 
In the work of Yang et al. (2010), a steady-state-based approach was proposed which can be used to detect small actuator stuck failures including a zero value. Based on the approach, a dynamic output feedback controller was designed for both fault-free and post-fault systems. The work Wu et al. (2014), non-parameterizable bounded time-varying stuck failures were assumed to be piecewise continuous bounded functions and they were compensated by a robust adaptive fault-tolerant controller. Jiang and Chowdhury (2005) proposed a methodology for detection and accommodation of multi-type faults and stuck failures. An active FTC approach was used and extended to a system with an unknown input.

However, the drawback of these approaches is the lack of considering actuator constraints. Without these, the available control inputs of a post-fault system are infinite, which implies that the system has infinite redundancy. On the other hand, with actuator constraints and an actuator stuck failure, the effective ability of the other actuators to remain failure-free is decreased because some effectiveness is used to compensate a constant control input and the lost control effectiveness caused by the stuck actuator. Under this condition, actuator saturation is more likely to occur. To avoid saturation, locally bounded controller outputs of a post-fault system were considered by Jiang et al. (2006) through appropriate assumptions. $\mathrm{Xu}$ et al. (2015) used a backstepping fault-tolerant controller with a command filter against both actuator faults and stuck failures under actuator constraints for a near-space vehicle. Moreover, in order to maintain admissible degradation performance, new references will be required if the original one is not reachable. Taking set-point reference, for example, unreachability means that actuators are saturated in steady-state under this reference.

In the work of Dardinier-Maron et al. (1999), a new reference of post-fault system was generated according to the system's remaining performance. The distance between the new reference and the original one before failure is minimum. Zhang and Jiang (2003) investigated a control input management approach to compute a new steady-state reference which is based on the open-loop gain of a post-fault system in the steady case. The tuned experimentally so that the new reference may not be optimal.

In the work of Theilliol et al. (2008), a model predictive control strategy was proposed to redesign the new reference on-line which was achieved by solving an optimization problem, but only the reduction in effectiveness of actuators was considered. The issue neglected by these approaches is that not all faults can be compensated when system performance is limited. It is necessary to analyze the compensability of actuator faults depending on the remaining systems performance. What is more serious, if a system subject to actuator constraints is open-loop unstable, only regional stability can be guaranteed (Wu and Soto, 2003). The approaches above cannot provide the stability region of a post-fault system which is open-loop unstable.

In this paper, actuator stuck failures and asymmetrical actuator constraints are considered simultaneously subject to an open-loop unstable system. As the main contribution, actuator stuck failures are originally defined as actuator constraints so that a new fault-free system with new actuator constraints is constructed. Based on the new system, the controller is reconfigured to achieve failure accommodation. Compared with the conventional FTC structure, an extended control framework is developed to guarantee post-fault system safety. As shown in Fig. 1, the framework consists of three parts: a reconfigurable controller, an evaluator and reference redesign module. The evaluator module is used to analyze the performance of post-fault system based on failure information and the original reference input. If the reference is unreachable, reference redesign module will compute a new optimal one to guarantee system safety. The required information of actuator stuck failures is supposed to be provided correctly by an FDI module. The design of the FDI module is beyond the scope of this article and related methods can be found in various contributions (e.g., Yang et al., 2010; Chen and Patton, 2012; Ossmann and Varga, 2015).

The paper is organized as follows. In Section 2 definitions of actuator stuck failures and actuator constraints are reviewed. Then, the design objectives of this paper are presented. Section 3 discusses the main results in two parts, including gain synthesis and set-point tracking. Simulation samples are shown in Section 4 Section 5 concludes the paper.

\section{Problem statement}

Consider a system as the following expression:

$$
\left\{\begin{array}{l}
\dot{x}(t)=A x(t)+B \operatorname{sat}(u(t)) \\
y(t)=C x(t)
\end{array}\right.
$$

where $x \in \mathbb{R}^{n}$ is the system state vector, $u \in \mathbb{R}^{m}$ is the actuator output vector in absence of constraints, $y \in \mathbb{R}^{p}$ is the system controlled output vector, $A, B, C$ are constant matrices with appropriate dimensions and $\operatorname{sat}(\cdot)$ is a nonlinear function defined by

$$
\operatorname{sat}\left(u_{i}(t)\right)= \begin{cases}u_{i}^{\max }, & u_{i}(t)>u_{i}^{\max } \\ u_{i}(t), & u_{i}^{\min } \leq u_{i}(t) \leq u_{i}^{\max } \\ u_{i}^{\min }, & u_{i}(t)<u_{i}^{\min }\end{cases}
$$

where $i=1, \ldots, m, u^{\min }$ and $u^{\max }$ signify constant actuator constraints. For convenience, we set $u_{i}^{\max }=$ 


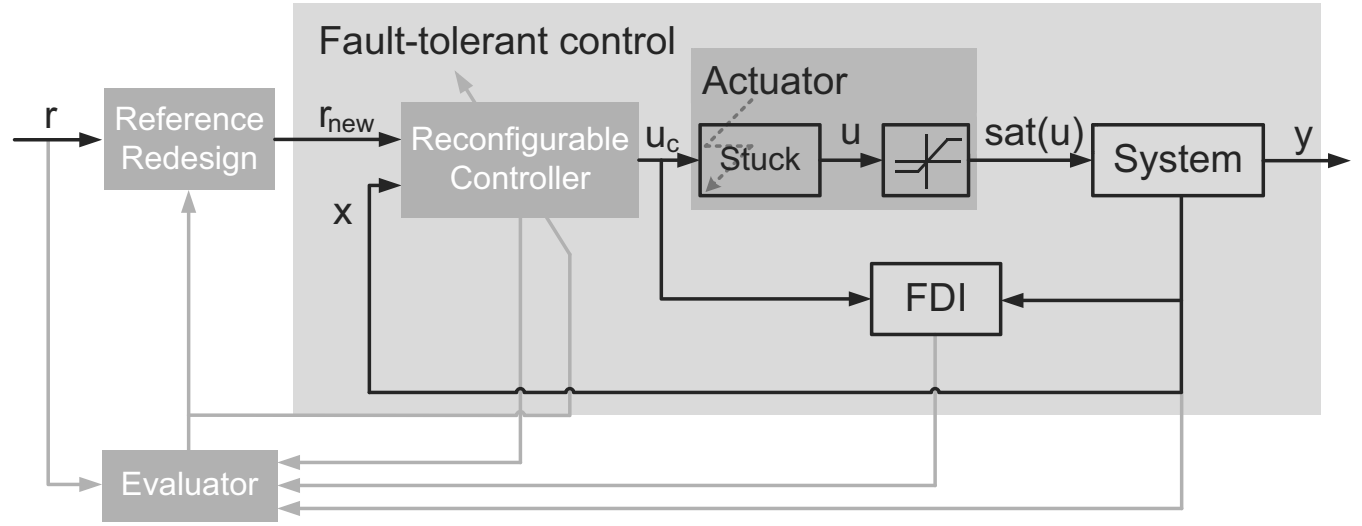

Fig. 1. Proposed control framework.

$\alpha_{i}, u_{i}^{\min }=-\beta_{i}$ and $\alpha=\operatorname{diag}\left[\alpha_{1}, \alpha_{2}, \ldots, \alpha_{m}\right]$, $\beta=\operatorname{diag}\left[\beta_{1}, \beta_{2}, \ldots, \beta_{m}\right]$. Note that $\alpha_{i}=\beta_{i}$ represents the fact that the constraints of the $i$-th actuator are symmetrical and $\alpha_{i} \neq \beta_{i}$ stands for asymmetrical constraints. These are considered in the following analysis to generalize the proposed method.

Actuator stuck failures can be modeled as

$$
u(t)=\Gamma u_{c}(t)+\left(I_{m \times m}-\Gamma\right) \bar{u},
$$

where $u_{c}(t)$ represents the controller output vector and $\bar{u}$ is a constant vector. $\Gamma=\operatorname{diag}\left(\gamma_{1}, \gamma_{2}, \ldots, \gamma_{m}\right)$ and $\gamma_{i}=1$ or 0 , where $\gamma_{i}=1$ means that the $i$-th actuator is fault-free and $\gamma_{i}=0$ represents the fact that the $i$-th actuator is lock-in-place so that the actuator cannot respond the control signal $u_{c}(t)$.

Hence, the actuator output vector in the absence of constraints can be divided into two parts: $u(t)=$ $\left[u_{0}^{T}(t) \bar{u}_{f}^{T}\right]^{T}$ in a stuck-failure case, where $u_{0}(t) \in \mathbb{R}^{m_{0}}$ is the fault-free actuator output, $\bar{u}_{f} \in \mathbb{R}^{m_{f}}$ is the stuck actuator output and $m_{0}+m_{f}=m$. The control matrix $B$ can be decomposed into $\left[B_{0} B_{f}\right]$ with $B_{0} \in \mathbb{R}^{n \times m_{0}}$ and $B_{f} \in \mathbb{R}^{n \times m_{f}}$.

In the following discussion, assume that the first $m_{0}$ actuators are fault-free and the last $m_{f}$ actuators are stuck. Then the system (1) in the stuck-failure case can be described as

$$
\left\{\begin{array}{l}
\dot{x}(t)=A x(t)+B_{0} \operatorname{sat}\left(u_{0}(t)\right)+B_{f} \bar{u}_{f}, \\
y(t)=C x(t)
\end{array}\right.
$$

The following discussion will be based on the system (2), which satisfies two assumptions.

Assumption 1. The post-fault system (2) is globally stable in the absence of actuator constraints.

Assumption 2. $\operatorname{rank}(A)=n$.

Specifically, an open-loop unstable system is considered in the paper. For an open-loop unstable system under actuator constraints, global stability cannot be guaranteed (Wu and Soto, 2003), so that regional stability has to be considered. At the same time, actuator stuck failures can affect the stability region by reducing the number of available control inputs and bringing a constant control input. Both actuator stuck failures and constraints should be taken into account when calculating regions so that unifying failures and constraints will simplify the problem of controller synthesis and performance analysis. Simultaneously, the analysis of stuck-failure compensability and reference reachability is also related to stability regions. Consequently, the following problems should be solved to provide scientific contributions of the paper:

Problem 1. Find a solution to unify actuator stuck failures and constraints, because they are coupled due to actuators and both of them can affect the stability region.

Problem 2. Design a fault-tolerant controller to guarantee post-fault system regional stability and analyze the compensability of actuator stuck failures before fault-tolerant controller switches.

Problem 3. Design a fault-tolerant and set-point tracking controller and analyze reference reachability before controller switches. If the original one is unreachable, compute a new optimal reference for the system.

\section{Main results}

3.1. Gain synthesis problem against actuator stuck failures under constraints. Due to the mutual influence of actuator stuck failures and constraints, both of these factors must be considered at the same time. In order to unify them, actuator stuck failures are defined as actuator constraints.

Recall the state equation of the post-fault system (2),

$$
\dot{x}(t)=A x(t)+B_{0} \operatorname{sat}\left(u_{0}(t)\right)+B_{f} \bar{u}_{f} .
$$


The constant control input $\bar{u}_{f}$ caused by a stuck actuator, Fig. 2(a), can be described as

$$
\bar{u}_{f}=u_{f}(t)+\omega(t),
$$

where $u_{f}(t) \in \mathbb{R}^{m_{f}}$ and $\omega(t) \in \mathbb{R}^{m_{f}}$ satisfy the following relationship, as shown in Fig. 2(b).

$$
\begin{gathered}
\bar{u}_{f}-\varepsilon \leq u_{f}(t) \leq \bar{u}_{f}+\varepsilon, \\
-\varepsilon \leq \omega(t) \leq \varepsilon,
\end{gathered}
$$

where $\varepsilon \in \mathbb{R}^{m_{f}}$ and $\varepsilon=\left[\varepsilon_{m_{0}+1}, \varepsilon_{m_{0}+2}, \ldots, \varepsilon_{m_{f}}\right] \geq 0$.

Note that $\beta_{f}=\operatorname{diag}\left[-\left(\bar{u}_{f}-\varepsilon\right)\right]$ and $\alpha_{f}=\operatorname{diag}\left[\bar{u}_{f}+\right.$ $\varepsilon]$ are lower and upper bounds of $u_{f}(t)$ so that $u_{f}(t)$ can be described as sat $\left(u_{f}(t)\right)$. The state equation (3) is rewritten as

$$
\begin{aligned}
\dot{x}(t) & =A x(t)+\left[\begin{array}{ll}
B_{0} & B_{f}
\end{array}\right]\left\{\left[\begin{array}{l}
\operatorname{sat}\left(u_{0}(t)\right) \\
\operatorname{sat}\left(u_{f}(t)\right)
\end{array}\right]+\left[\begin{array}{c}
0 \\
\omega(t)
\end{array}\right]\right\} \\
& =A x(t)+B \operatorname{sat}(\tilde{u}(t))+B_{f} \omega(t),
\end{aligned}
$$

with new constraints $\tilde{\alpha}=\operatorname{diag}\left[\alpha_{0} \quad \alpha_{f}\right]$ and $\tilde{\beta}=$ $\operatorname{diag}\left[\beta_{0} \beta_{f}\right]$, where $\alpha_{0}$ and $\beta_{0}$ are bounds of the remaining fault-free actuators. Obviously, if $\bar{u}_{f}$ is non-zero, the constraints of $u_{f}(t)$ will be asymmetrical.

To the best of our knowledge, this method, which defines actuator stuck failures as actuator constraints, has never been investigated before and is firstly proposed in this paper.

Asymmetrical constraints can be transformed into symmetrical ones according to the following equation, as proposed by Benhayoun et al. (2013).

$$
\operatorname{sat}(\tilde{u}(t))=\frac{\tilde{\alpha}+\tilde{\beta}}{2} \operatorname{sat}(v(t))+\frac{\tilde{\alpha}-\tilde{\beta}}{2} \zeta,
$$

where the control input $v(t)$ satisfies $-1 \leq v(t) \leq 1$, and $\zeta$ is a unit vector. Clearly, the constraints of $v(t)$ are symmetrical, as shown in Fig. 2(c).

Then the state equation can be described as

$$
\begin{aligned}
\dot{x}(t)= & A x(t)+B \frac{\tilde{\alpha}+\tilde{\beta}}{2} \operatorname{sat}(v(t)) \\
& +B \frac{\tilde{\alpha}-\tilde{\beta}}{2} \zeta+B_{f} \frac{\alpha_{f}+\beta_{f}}{2} \tilde{\omega}(t) \\
= & A x(t)+\tilde{B} \operatorname{sat}(v(t))+\tilde{B}_{f} \tilde{\omega}(t)+B \frac{\tilde{\alpha}-\tilde{\beta}}{2} \zeta,
\end{aligned}
$$

where $-1 \leq v(t) \leq 1,-1 \leq \tilde{\omega}(t) \leq 1$,

$$
\tilde{B}=B \frac{\tilde{\alpha}+\tilde{\beta}}{2}, \quad \tilde{B}_{f}=B_{f} \frac{\alpha_{f}+\beta_{f}}{2} .
$$

In order to remove the constant term $B \frac{\tilde{\alpha}-\tilde{\beta}}{2} \zeta$ from (9), a new state is defined as

$$
\tilde{x}(t)=x(t)+A^{-1} B \frac{\tilde{\alpha}-\tilde{\beta}}{2} \zeta
$$

Then a new state equation of an open-loop system, which is equal to (3), is obtained,

$$
\dot{\tilde{x}}(t)=A \tilde{x}(t)+\tilde{B} \operatorname{sat}(v(t))+\tilde{B}_{f} \tilde{\omega}(t) .
$$

Note that, compared with the system (3), the system (12) only has actuator constraints but no actuator stuck failures. It should also be pointed out that the stability of the system (12) is a sufficient conditions of the stability of the system (3). Thus, actuator stuck failures and constraints are unified, and Problem 1 has been solved.

Then, considering the system (12) with state-feedback controller $v(t)=K \tilde{x}(t)$,

$$
\dot{\tilde{x}}(t)=A \tilde{x}(t)+\tilde{B} \operatorname{sat}(K \tilde{x}(t))+\tilde{B}_{f} \tilde{\omega}(t),
$$

the gain matrix $K$ can be determined by the following theorem to guarantee regional stability and prevent actuators from saturation.

Theorem 1. Given $\varepsilon \geq 0, \eta>0$, if there exists a symmetric positive-definite matrix $W \in \mathbb{R}^{n \times n}$, a matrix $Y \in \mathbb{R}^{m \times n}$ satisfies

$$
\left[\begin{array}{cc}
1 & Y_{i} \\
* & W
\end{array}\right] \geq 0, \quad i=1, \ldots, m
$$

$$
W A^{T}+A W+Y^{T} \tilde{B}^{T}+\tilde{B} Y+\frac{1}{\eta} \tilde{B}_{f} \tilde{B}_{f}^{T}+\eta W<0,
$$

where $Y_{i}$ denotes the $i$-th row of matrix $Y$; then the stability region of the system (13) can be represented by ellipsoidal invariant set $\Omega(P)=\left\{\tilde{x}(t) \in \mathbb{R}^{n} \mid \tilde{x}^{T}(t) P \tilde{x}(t) \leq\right.$ $1\}$, with $P=W^{-1}$. At the same time, actuators are never saturated for $\tilde{x}(t) \in \Omega(P)$, with $K=Y P$.

Proof. The satisfaction of relations (14) denotes $\Omega(P) \subseteq$ $\Xi(K)=\left\{\tilde{x}(t) \in \mathbb{R}^{n}|| K_{i} \tilde{x}(t) \mid \leq 1, \quad i=1, \ldots, m\right\}$, which implies that actuator constraints will be satisfied if $\tilde{x}(t) \in \Omega(P)$ (Hu et al., 2002). Considering a Lyapunov function $V(\tilde{x}(t))=\tilde{x}^{T}(t) P \tilde{x}(t)>0$ and $-1 \leq \tilde{\omega}(t) \leq$ 1 , its time-derivative is

$$
\begin{aligned}
\dot{V}(\tilde{x}(t))= & \tilde{x}^{T}(t)\left[(A+\tilde{B} K)^{T} P+P(A+\tilde{B} K)\right] \tilde{x}(t) \\
& +\tilde{\omega}^{T}(t) \tilde{B}_{f}^{T} P \tilde{x}(t)+\tilde{x}^{T}(t) P \tilde{B}_{f} \tilde{\omega}(t) \\
\leq & \tilde{x}^{T}(t)\left[(A+\tilde{B} K)^{T} P+P(A+\tilde{B} K)\right. \\
& \left.+\frac{1}{\eta} P \tilde{B}_{f} \tilde{B}_{f}^{T} P\right] \tilde{x}(t)+\eta \\
= & \tilde{x}^{T}(t)\left[(A+\tilde{B} K)^{T} P+P(A+\tilde{B} K)\right. \\
+ & \left.\frac{1}{\eta} P \tilde{B}_{f} \tilde{B}_{f}^{T} P+\eta P\right] \tilde{x}(t)-\eta \tilde{x}^{T}(t) P \tilde{x}(t)+\eta .
\end{aligned}
$$

Note that, on the boundary of $\Omega(P), \tilde{x}^{T}(t) P \tilde{x}(t)=1$. Hence $\dot{V}(\tilde{x}(t))<0$ if the following inequality is satisfied:

$$
(A+\tilde{B} K)^{T} P+P(A+\tilde{B} K)+\frac{1}{\eta} P \tilde{B}_{f} \tilde{B}_{f}^{T} P+\eta P<0 .
$$




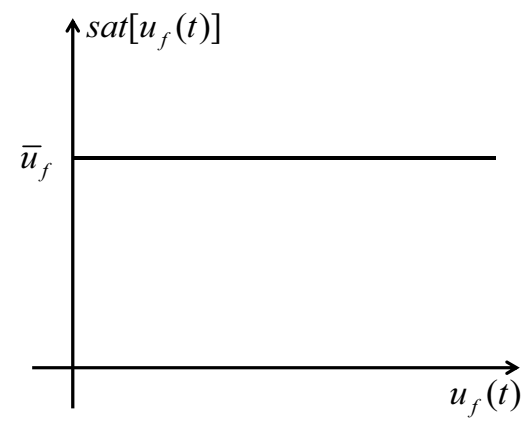

(a)

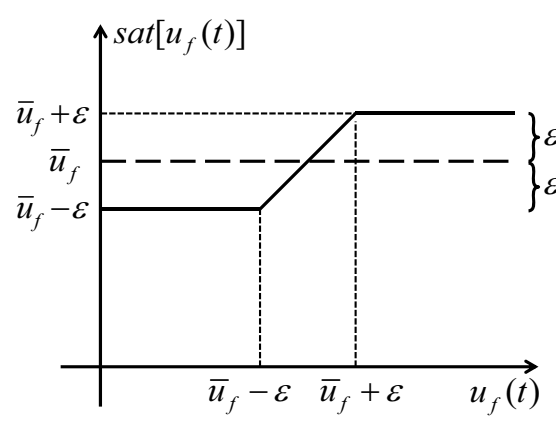

(b)

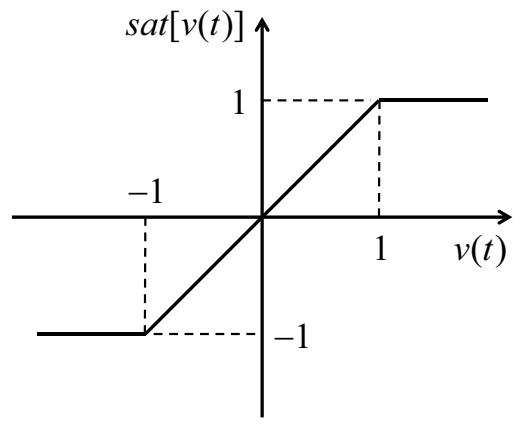

(c)

Fig. 2. Redefinition of the actuator stuck failure as actuator constraints: stuck failure (a), asymmetrical constraints (b), symmetrical constraints (c).

Then (15) is achieved by pre- and post-multiplying the above inequality by $P^{-1}$ and replacing $K P^{-1}$ with $Y$.

Thus, regional stability of the system (13) is guaranteed by stability region $\Omega(P)$. According to (11), the stability region of $x(t)$ is $\Omega_{x}(P)=\{x(t) \in$ $\mathbb{R}^{n} \mid\left(x(t)+A^{-1} B \frac{\tilde{\alpha}-\tilde{\beta}}{2} \zeta\right)^{T}(t) P\left(x(t)+A^{-1} B \frac{\tilde{\alpha}-\tilde{\beta}}{2} \zeta\right) \leq$ $1\}$. Obviously, $\Omega_{x}(P)$ and $\Omega(P)$ are ellipsoids with the same scale and shape, but their centers are different. If actuator constraints are symmetrical, the center of $\Omega(P)$ will be the origin and the one of $\Omega_{x}(P)$ will be $-A^{-1} B \frac{\tilde{\alpha}-\tilde{\beta}}{2} \zeta=-A^{-1} B\left[\begin{array}{ll}0 & \bar{u}_{f}^{T}\end{array}\right]^{T}$. Clearly, the center is related to stuck failure $\bar{u}_{f}$.

Remark 1. The system (12) is not affected by the value of stuck failure $\bar{u}_{f}$. Thus, the existing research results against actuator constraints are able to be used for stuck compensation analysis.

In order to employ Theorem 1 , the value of $\varepsilon$ should be selected first. The following theorem improves Theorem 1 and provides a way to fix $\varepsilon$ and the other variables of Theorem 1 together.

Theorem 2. Given $\eta>0$, if there exists a symmetric positive-definite matrix $W \in \mathbb{R}^{n \times n}$, a matrix $Y \in \mathbb{R}^{m \times n}$ and a set of positive scales $\epsilon=\left[\epsilon_{m_{0}+1}, \epsilon_{m_{0}+1}, \ldots, \epsilon_{m_{f}}\right]$ satisfying

$$
\begin{gathered}
{\left[\begin{array}{cc}
1 & Y_{i} \\
* & W
\end{array}\right] \geq 0, \quad i=1, \ldots, m_{0},} \\
{\left[\begin{array}{cc}
\epsilon_{j} & Y_{j} \\
* & W
\end{array}\right] \geq 0, \quad j=m_{0}+1, \ldots, m_{f},} \\
W A^{T}+A W+Y^{T} \bar{B}^{T}+\bar{B} Y \\
+\frac{1}{\eta} B_{f}\left[\begin{array}{ccc}
\epsilon_{m_{0}+1} & 0 \\
0 & \ddots & \epsilon_{m_{f}}
\end{array}\right] B_{f}^{T}+\eta W<0,
\end{gathered}
$$

then the ellipsoid $\Omega(P)=\left\{\tilde{x}(t) \in \mathbb{R}^{n} \mid \tilde{x}^{T}(t) P \tilde{x}(t) \leq 1\right\}$ is an invariant set and satisfies $\Omega(P) \subseteq \Xi(K)$, where $\varepsilon_{i}=\sqrt{\epsilon_{i}} i=m_{0}+1, \ldots, m_{f}, P=W^{-1}, Y_{i}, Y_{j}$ represent the $i$-th, $j$-th rows of matrix $Y$, and

$$
K=\left[\begin{array}{cccc}
I_{m_{0} \times m_{0}} & & & 0 \\
& \frac{1}{\varepsilon_{m_{0}+1}} & & \\
0 & & \ddots & \\
0 & & & \frac{1}{\varepsilon_{m_{f}}}
\end{array}\right] Y P .
$$

Proof. Consider Eqn. (10),

$$
\begin{aligned}
& \tilde{B}=B \frac{\tilde{\alpha}+\tilde{\beta}}{2} \\
&=B\left[\begin{array}{cc}
\frac{\alpha_{0}+\beta_{0}}{2} & 0 \\
0 & I_{m_{f} \times m_{f}}
\end{array}\right]\left[\begin{array}{cc}
I_{m_{0} \times m_{0}} & 0 \\
0 & \operatorname{diag}[\varepsilon]
\end{array}\right] \\
&=\bar{B}\left[\begin{array}{cc}
I_{m_{0} \times m_{0}} & 0 \\
0 & \operatorname{diag}[\varepsilon]
\end{array}\right], \\
& \tilde{B}_{f}=B_{f} \frac{\alpha_{f}+\beta_{f}}{2}=B_{f} \operatorname{diag}[\varepsilon] .
\end{aligned}
$$

Thus, the condition (15) of Theorem 1 can be rewritten as

$$
\begin{aligned}
W & A^{T}+A W+Y^{T} \tilde{B}^{T}+\tilde{B} Y+\frac{1}{\eta} \tilde{B}_{f} \tilde{B}_{f}^{T}+\eta W \\
= & W A^{T}+A W+Y^{T}\left[\begin{array}{cc}
I_{m_{0} \times m_{0}} & 0 \\
0 & \operatorname{diag}[\varepsilon]
\end{array}\right] \bar{B}^{T} \\
& +\bar{B}\left[\begin{array}{cc}
I_{m_{0} \times m_{0}} & 0 \\
0 & \operatorname{diag}[\varepsilon]
\end{array}\right] Y \\
& +\frac{1}{\eta} B_{f} \operatorname{diag}[\varepsilon] \operatorname{diag}[\varepsilon] B_{f}^{T}+\eta W \\
= & W A^{T}+A W+\bar{Y}^{T} \bar{B}^{T}+\bar{B} \bar{Y} \\
& +\frac{1}{\eta} B_{f}\left[\begin{array}{cc}
\varepsilon_{m_{0}+1}^{2} & 0 \\
0 & \ddots
\end{array}\right] B_{f}^{T}+\eta W<0,
\end{aligned}
$$


with

$$
\bar{Y}=\left[\begin{array}{cc}
I_{m_{0} \times m_{0}} & 0 \\
0 & \operatorname{diag}[\varepsilon]
\end{array}\right] Y .
$$

In order to guarantee $|K \tilde{x}(t)| \leq 1, \bar{K}=\bar{Y} P$ and $|\bar{K} \tilde{x}(t)| \leq\left[I_{1 \times m_{0}}, \varepsilon\right]^{T}$ should be satisfied which amount to

$$
\begin{gathered}
{\left[\begin{array}{cc}
1 & \bar{Y}_{i} \\
* & W
\end{array}\right] \geq 0, \quad i=1, \ldots, m_{0}} \\
{\left[\begin{array}{cc}
\varepsilon_{j}^{2} & \bar{Y}_{j} \\
* & W
\end{array}\right] \geq 0, \quad j=m_{0}+1, \ldots, m_{f} .}
\end{gathered}
$$

Thus, replace $\bar{Y}$ and $\left[\varepsilon_{m_{0}+1}^{2}, \varepsilon_{m_{0}+2}^{2}, \ldots, \varepsilon_{m_{f}}^{2}\right]$ with $Y$ and $\epsilon=\left[\epsilon_{m_{0}+1}, \epsilon_{m_{0}+2}, \ldots, \epsilon_{m_{f}}\right]$, respectively, and Theorem 2 is proved.

According to the stability region of a post-fault system, failure compensability can be analyzed before fault-tolerant controller switches. Assume that the stuck failure $\bar{u}_{f}$ occurs at instant $t_{u}$, and it is detected and isolated at instant $t_{f}$. Note that the time delay between $t_{u}$ and $t_{f}$ is due to the FDI module, and before the stuck failure is detected and isolated, the system is also controlled by the fault-free controller. Thus, the fault-tolerant controller will replace the fault-free one at instant $t_{f}$. Define the related system states $x\left(t_{f}\right)$ at instant $t_{f}$. Because all of the states in $\Omega_{x}(P)$ can guarantee the stability of a post-fault system and $\Omega_{x}(P)$ is an invariant set, the stuck failure can be compensated by the new controller despite FDI delay if

$$
x\left(t_{f}\right) \in \Omega_{x}(P) .
$$

In other words, if $x\left(t_{f}\right)$ is involved in the stability region $\Omega_{x}(P)$, the post-fault system will be stabilized by the redesigned controller through Theorem 2 under actuator stuck failure $\bar{u}_{f}$ and actuator constraints. Furthermore, because $\Omega_{x}(P)$ is an invariant set, if the initial state of post-fault system, $x\left(t_{f}\right)$, is inside the set, the post-fault system will be stable as $t \rightarrow \infty$. Hence, before controller switches, the failure compensability can be analyzed. Thus, a solution to Problem 2 is found.

Note that the proposed method is just interested in $x\left(t_{f}\right)$, and FDI time delay has no influence on the proposed method itself. On the other hand, if the time delay is too large, the post-fault system may be out of order. The longest allowed FDI time delay can be calculated as described by Tabatabaeipour and Blanke (2014).

Remark 2. The stability region $\Omega_{x}(P)$ calculated by Theorems 1 and 2 is a subset of the actual stability region because the stability criterion used in Theorem 1 and 2 is a sufficient condition and the ellipsoid invariant set is conservative. Thus, $x\left(t_{f}\right) \in \Omega_{x}(P)$ is also a sufficient condition for which the stuck failure $\bar{u}_{f}$ is compensable. Under this condition, the stability region $\Omega_{x}(P)$ should be enlarged to decrease the conservative property and some methods have been proposed to enlarge or even obtain the largest region (da Silva and Tarbouriech, 2005; Li and Lin, 2013; Hu et al., 2002).

3.2. Set-point tracking against actuator stuck failures and constraints. The control objective is to guarantee the stability of the post-fault system with acceptable set-point tracking performance. Consider the system (2) with integrator $e(t)$ as follows:

$$
\left\{\begin{array}{l}
\dot{x}(t)=A x(t)+B_{0} \operatorname{sat}\left(u_{0}(t)\right)+B_{f} \bar{u}_{f}, \\
y(t)=C x(t) \\
\dot{e}(t)=r-y(t)
\end{array}\right.
$$

where $e(t)=\int(r-C x(t)) \mathrm{d} t \in \mathbb{R}^{p}$, and $r \in \mathbb{R}^{p}$ is a set-point reference with constraint $r^{T} r \leq \rho, \rho>0$.

According to (4)-(11), the transformed open-loop system without a stuck failure is constructed,

$$
\left\{\begin{array}{l}
\dot{\tilde{x}}(t)=A \tilde{x}(t)+\tilde{B} \operatorname{sat}(v(t))+\tilde{B}_{f} \tilde{\omega}(t), \\
\tilde{y}(t)=C \tilde{x}(t), \\
\dot{e}(t)=\tilde{r}-\tilde{y}(t)
\end{array}\right.
$$

Note that, according to 11, $\tilde{y}(t)=C \tilde{x}(t)=$ $C\left(x(t)+A^{-1} B \frac{\tilde{\alpha}-\tilde{\beta}}{2} \zeta\right)=y(t)+C A^{-1} B \frac{\tilde{\alpha}-\tilde{\beta}}{2} \zeta$. Thus, define the reference for the system (16) as $\tilde{r}=r+$ $C A^{-1} B \frac{\tilde{\alpha}-\tilde{\beta}}{2} \zeta$. Consider a state feedback controller

$$
v(t)=K \tilde{x}(t)+K_{r} e(t),
$$

where $K$ and $K_{r}$ are pending controller matrices. Hence, neglecting actuator saturation, the closed-loop system is described as

$$
\left\{\begin{array}{l}
\dot{\xi}(t)=(\mathcal{A}+\mathcal{B} \mathcal{K}) \xi(t)+\mathcal{B}_{f} \tilde{\omega}(t)+\mathcal{H} \tilde{r}, \\
\tilde{y}(t)=\mathcal{C} \xi(t)
\end{array}\right.
$$

with an extended state vector $\xi(t)=\left[\tilde{x}^{T}(t) e^{T}(t)\right]^{T} \in$ $\mathbb{R}^{n+p}$ and the following matrices:

$$
\begin{gathered}
\mathcal{A}=\left[\begin{array}{cc}
A & 0 \\
-C & 0
\end{array}\right], \\
\mathcal{B}=\left[\begin{array}{c}
\tilde{B} \\
0
\end{array}\right]=\overline{\mathcal{B}}\left[\begin{array}{cc}
I_{m_{0} \times m_{0}} & 0 \\
0 & \operatorname{diag}[\varepsilon]
\end{array}\right], \\
\overline{\mathcal{B}}=\left[\begin{array}{c}
B \\
0
\end{array}\right]\left[\begin{array}{cc}
\frac{\alpha_{0}+\beta_{0}}{2} & 0 \\
0 & I_{m_{f} \times m_{f}}
\end{array}\right], \\
\mathcal{B}_{f}=\left[\begin{array}{c}
\tilde{B}_{f} \\
0
\end{array}\right]=\overline{\mathcal{B}}_{f} \operatorname{diag}[\varepsilon], \\
\overline{\mathcal{B}}_{f}=\left[\begin{array}{c}
B_{f} \\
0
\end{array}\right], \quad \mathcal{H}=\left[\begin{array}{l}
0 \\
I
\end{array}\right], \\
\mathcal{C}=[C 0], \quad \mathcal{K}=\left[K K_{r}\right] .
\end{gathered}
$$


In order to guarantee the stability of the closed-loop system and prevent saturation of actuators, the following theorem is proposed.

Theorem 3. Given $\eta>0, \rho>0$, if there exists a symmetric positive-definite matrix $W \in \mathbb{R}^{(n+p) \times(n+p)}$, a matrix $Y \in \mathbb{R}^{m \times(n+p)}$, a set of positive scales $\epsilon=$ $\left[\epsilon_{m_{0}+1}, \epsilon_{m_{0}+1}, \ldots, \epsilon_{m_{f}}\right]$, and a positive scale $\gamma$ satisfying

$$
\begin{gathered}
{\left[\begin{array}{ll}
1 & Y_{i} \\
* & W
\end{array}\right] \geq 0, \quad i=1, \ldots, m_{0},} \\
{\left[\begin{array}{cc}
\epsilon_{j} & Y_{j} \\
* & W
\end{array}\right] \geq 0, \quad j=m_{0}+1, \ldots, m_{f},} \\
{\left[\begin{array}{ccc}
Q_{1} & \mathcal{H} & -W \mathcal{C}^{T} \\
* & -\gamma \rho I & I \\
* & * & -I
\end{array}\right]<0,}
\end{gathered}
$$

where

$$
\begin{aligned}
Q_{1}= & W \mathcal{A}^{T}+\mathcal{A} W+Y^{T} \overline{\mathcal{B}}^{T}+\overline{\mathcal{B}} Y \\
& +\frac{1}{\eta} \overline{\mathcal{B}}_{f}\left[\begin{array}{ccc}
\epsilon_{m_{0}+1} & & 0 \\
& \ddots & \\
0 & & \epsilon_{m_{f}}
\end{array}\right] \overline{\mathcal{B}}_{f}^{T}+\eta W,
\end{aligned}
$$

$Y_{i}$ and $Y_{j}$ represent the $i$-th and $j$-th rows of matrix $Y$, respectively then the ellipsoid $\Omega(P)=\{\xi(t) \in$ $\left.\mathbb{R}^{(n+p)} \mid \xi^{T}(t) P \xi(t) \leq 1\right\}$ is an invariant set and satisfies $\Omega(P) \subseteq \Xi(K)$ with $\varepsilon_{i}=\sqrt{\epsilon_{i}} i=m_{0}+1, \ldots, m_{f}$, $P=W^{-1}$ and

$$
\mathcal{K}=\left[\begin{array}{cccc}
I_{m_{0} \times m_{0}} & & & 0 \\
& \frac{1}{\varepsilon_{m_{0}+1}} & & \\
0 & & \ddots & \\
& & & \frac{1}{\varepsilon_{m_{f}}}
\end{array}\right] Y P
$$

In other words, the region $\Omega(P)$ is the stability region of the system (18) that guarantees the absence of actuator saturation and tracking performance.

Proof. Considering a Lyapunov function of the system (18), $V(\xi(t))=\xi^{T}(t) P \xi(t)$, according to Theorem 1, the derivative satisfies

$$
\dot{V}(\xi(t)) \leq\left[\begin{array}{c}
\xi(t) \\
\tilde{r}
\end{array}\right]^{T}\left[\begin{array}{cc}
Q_{2} & P \mathcal{H} \\
* & 0
\end{array}\right]\left[\begin{array}{c}
\xi(t) \\
\tilde{r}
\end{array}\right],
$$

where

$$
\begin{aligned}
Q_{2}= & \mathcal{A}^{T} P+P \mathcal{A}+\mathcal{K}^{T} \mathcal{B}^{T} P+P \mathcal{B} \mathcal{K} \\
& +\frac{1}{\eta} P \mathcal{B}_{f} \mathcal{B}_{f}^{T} P+\eta P
\end{aligned}
$$

Taking into account energy-to-energy performance $\|\dot{e}(t)\|_{2}=\|\tilde{r}-\mathcal{C} \xi(t)\|_{2} \leq \bar{\gamma}\|\tilde{r}\|_{2}$, and matrices $\overline{\mathcal{B}}$ and
$\overline{\mathcal{B}}_{f}$, the following relationship should be satisfied (Pascal and Pierre, 1994):

$$
\left[\begin{array}{ccc}
Q_{3} & P \mathcal{H} & -\mathcal{C}^{T} \\
* & -\bar{\gamma}^{2} \rho I & I \\
* & * & -I
\end{array}\right]<0
$$

where

$$
\begin{aligned}
Q_{3}= & \mathcal{A}^{T} P+P \mathcal{A}+\mathcal{K}^{T}\left[\begin{array}{cc}
I_{m_{0} \times m_{0}} & 0 \\
0 & \operatorname{diag}[\varepsilon]
\end{array}\right] \overline{\mathcal{B}}^{T} P \\
& +P \overline{\mathcal{B}}\left[\begin{array}{cc}
I_{m_{0} \times m_{0}} & 0 \\
0 & \operatorname{diag}[\varepsilon]
\end{array}\right] \mathcal{K} \\
& +\frac{1}{\eta} P \overline{\mathcal{B}}_{f} \operatorname{diag}[\varepsilon] \operatorname{diag}[\varepsilon] \overline{\mathcal{B}}_{f}^{T} P+\eta P .
\end{aligned}
$$

Then (21) is achieved by pre- and post-multiplying the inequality by

$$
\left[\begin{array}{ccc}
P^{-1} & 0 & 0 \\
0 & I & 0 \\
0 & 0 & I
\end{array}\right]
$$

and replacing $P^{-1}$,

$$
\left[\begin{array}{cc}
I_{m_{0} \times m_{0}} & 0 \\
0 & \operatorname{diag}[\varepsilon]
\end{array}\right] \mathcal{K} P^{-1},
$$

$\left[\varepsilon_{m_{0}+1}^{2}, \varepsilon_{m_{0}+2}^{2}, \ldots, \varepsilon_{m_{f}}^{2}\right]$ and $\bar{\gamma}^{2}$ by $W, Y, \epsilon=\left[\epsilon_{m_{0}+1}\right.$, $\left.\epsilon_{m_{0}+2}, \ldots, \epsilon_{m_{f}}\right]$ and $\gamma$, respectively. Thus, Theorem 3 is proved while the proof of (19) and 20) is the same as for Theorem 2.

In terms of the perspective of the task, the post-fault system is expected to track the original reference. However, the reachability of the original reference cannot be guaranteed by the post-fault system because of the existence of actuator constraints and actuator failures. Thus, in order to guarantee system safety, it is significant to evaluate the reference before controller switches. At the same time, if the original reference is unreachable for the post-fault system, a new optimal one will be required to maintain admissible degradation performance or at last keep system safety. Here, the optimal reference is defined as the one which is closest to the original one under the Euclidean distance and satisfies system constraints.

Considering the closed-loop system (18) and the relationship between $r$ and $\tilde{r}$, which is $\tilde{r}=r+$ $C A^{-1} B \frac{\tilde{\alpha}-\tilde{\beta}}{2} \zeta$, system states in a steady case can be calculated by the following equations for a specific 
set-point reference:

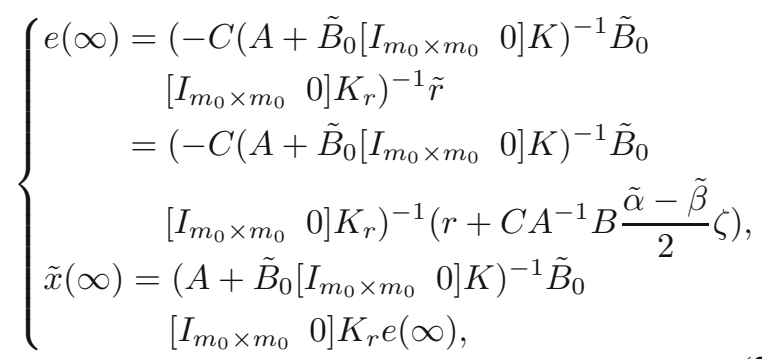

where $m_{0}$ is the number of fault-free actuators.

Then $\xi(\infty)=\left[\begin{array}{ll}\tilde{x}^{T}(\infty) & e^{T}(\infty)\end{array}\right]^{T}$ is achieved and $\xi(\infty)$ can be regarded as a function of $r$. According to the condition

$$
\xi(\infty)^{T} P \xi(\infty) \leq 1,
$$

the reachability of the original reference can be evaluated.

Obviously, if the above condition is satisfied, state $\xi(\infty)$ will be inside the stability region $\Omega(P)$. Furthermore, $x(\infty)$ is inside its stability region so that the reference is reachable. Hence, before controller switches, the reachability of the original reference can be evaluated. On the other hand, if reachability of the latter is not met, the original reference may lead to a post-fault system that is out of order. Hence a new reference, $r_{\text {new }}$, is required. From the perspective of maintaining admissible degradation performance, the new set-point reference should be as close as possible to the original one. Thus, the following theorem is proposed to find a new reference.

Theorem 4. Given a positive definite diagonal matrix $N \in \mathbb{R}^{p \times p}$ and a positive scale $\rho$, if there exists a vector $r_{\text {new }} \in \mathbb{R}^{p}$ and a positive scale $\lambda$ satisfying

$$
\begin{array}{cc}
\min _{r_{\text {new }}} \lambda \\
& {\left[\begin{array}{cc}
\lambda & \left(r_{\text {new }}-r\right)^{T} N \\
* & I
\end{array}\right] \geq 0,} \\
{\left[\begin{array}{cc}
1 & \left(Q\left(r+C A^{-1} B \frac{\tilde{\alpha}-\tilde{\beta}}{2} \zeta\right)\right)^{T} \\
* &
\end{array}\right] \geq 0,} \\
& {\left[\begin{array}{cc}
\rho & r^{-1} T \\
* & I
\end{array}\right] \geq 0,}
\end{array}
$$

$r_{\text {new }}$ is achieved as the optimal new reference.

Proof. In order to reduce the difference between the original reference $r$ and the new one $r_{\text {new }}$, the value of $\left\|N\left(r_{\text {new }}-r\right)\right\|_{2}$ should be minimized, which is equivalent to

$$
\begin{gathered}
\min _{r_{\text {new }}} \lambda \\
\left(r_{\text {new }}-r\right)^{T} N^{T} N\left(r_{\text {new }}-r\right) \leq \lambda,
\end{gathered}
$$

where $N$ is a known matrix to adjust weights of different elements in the new reference frame. The new element with a higher weight is closer to the original one. Considering the Schur complement, the inequality above can be described as 23).

In order to guarantee that $r_{\text {new }}$ is reachable by the post-fault system, according to (22), the following condition should be satisfied:

$$
\begin{array}{r}
\left(r+C A^{-1} B \frac{\tilde{\alpha}-\tilde{\beta}}{2} \zeta\right)^{T} Q^{T} P Q\left(r+C A^{-1} B \frac{\tilde{\alpha}-\tilde{\beta}}{2} \zeta\right) \\
\leq 1
\end{array}
$$

where

$$
\begin{aligned}
Q & =\left[\begin{array}{c}
M(-C M)^{-1} \\
(-C M)^{-1}
\end{array}\right], \\
M & =\left(\begin{array}{ll}
A+\tilde{B}_{0}\left[I_{m_{0} \times m_{0}}\right. & 0] K)^{-1} \tilde{B}_{0}\left[\begin{array}{ll}
I_{m_{0} \times m_{0}} & 0
\end{array}\right] K_{r} .
\end{array}\right.
\end{aligned}
$$

At the same time, $r_{\text {new }}$ should meet reference constraints such as $r_{\text {new }}^{T} r_{\text {new }} \leq \rho$.

Hence, according to the above two inequalities, 24 and 25) are achieved respectively by considering the Schur complement.

\section{Simulation samples}

In order to illustrate the proposed approaches and present simulation results more clearly, two numerical examples are given. The first one shows a different stability region, actuator outputs, and controller outputs between fault-free and post-fault systems. The second example is used for illustrating the set-point tracking approach against an actuator stuck failure and constraints.

\subsection{System stability of a fault-free and a post-fault} system. Consider the following unstable open-loop system:

$$
\dot{x}(t)=\left[\begin{array}{cc}
1 & -0.5 \\
1 & 3
\end{array}\right] x(t)+\left[\begin{array}{ll}
0 & 1 \\
2 & 0
\end{array}\right] \operatorname{sat}(u(t)) .
$$

The actuator constraints are $\alpha=\operatorname{diag}[21]$ and $\beta=$ $\operatorname{diag}\left[\begin{array}{ll}1 & 1\end{array}\right]$. For the fault-free condition, the controller $K$ and the related matrix $P$ are calculated by Theorem 1 with (15) being replaced by $W A^{T}+A W+Y^{T} \tilde{B}+\tilde{B} Y<0$. According to (11), the center of $\Omega_{x}(P)$, which is also the equilibrium point of the fault-free system, is $x_{0}=$ $-A^{-1} B \frac{\alpha-\beta}{2} \zeta=[-0.1429-0.2857]$. Simulation results are shown in Fig. 3 by solid lines with initial states $x(0)=[-0.7-0.5]$. Figure 3(a) shows the state space of $x(t)$ with state trajectory and stability regions. The initial states $x(0)$ are marked by the small circle and the final states are asymptotically converging to the star which is also the center of the real-line ellipse. Figure 3(b) shows actuator outputs of the system (26) and Fig. 3(c) shows the control inputs of the system (13). 


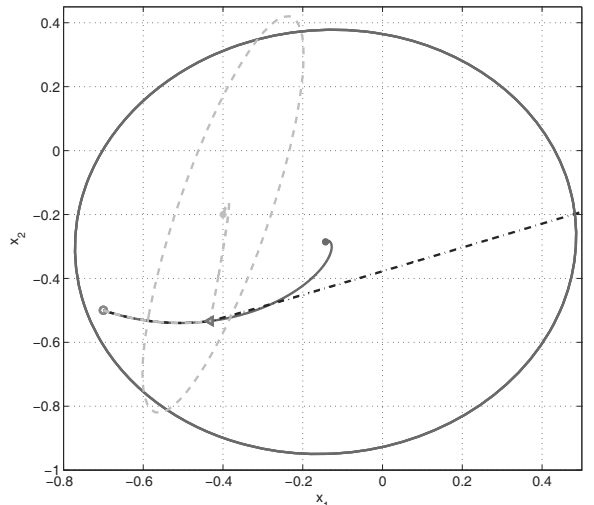

(a)
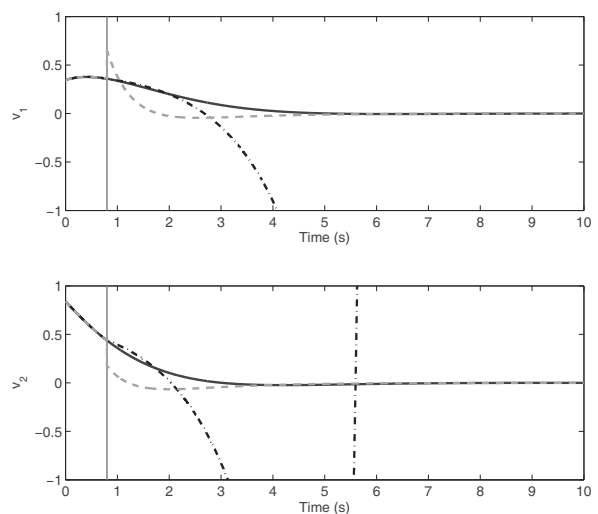

(c)
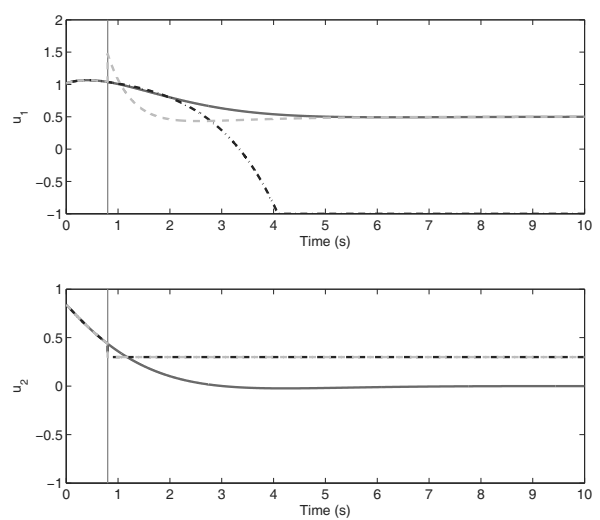

(b)

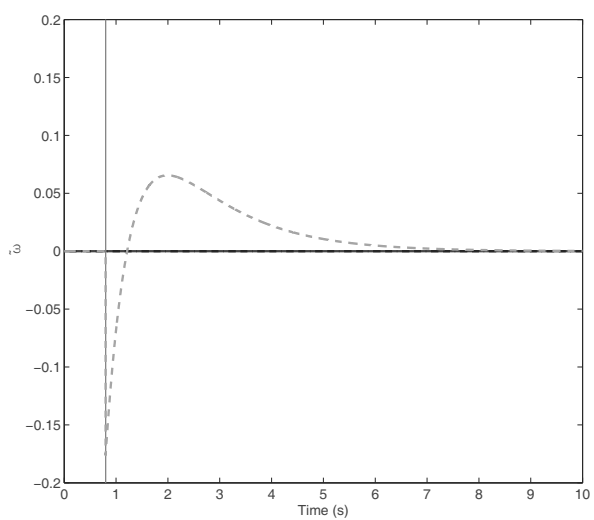

(d)

Fig. 3. Simulation results of a fault-free and a post-fault system: state space with stability regions and state trajectories (a), actuator outputs of the system (26) with the proposed controller (b), control inputs of the system (27) (c), $\tilde{\omega}(t)$ (d).

Assume the second actuator is stuck at $\bar{u}_{f}=0.3$ and detected at $t_{f}=0.8 \mathrm{~s}$, and the FDI module is supposed to provide fault information with no delay. As discussed above, the proposed method is only interested in $x\left(t_{f}\right)$, which is marked by a triangle in Fig. 3(a). Without the FTC approach, the post-fault system is out of order at last as shown in Fig. 3 by dot-dashed lines.

In order to guarantee the stability of the post-fault system, the controller should be redesigned. Taking into account the proposed control framework, the actuator stuck failure is redefined as constraints. Based on Theorem 2, $\varepsilon$ is calculated as 0.2060 with $\eta=1$. Thus, the new constraints achieved by (5) are $\tilde{\alpha}=\operatorname{diag}\left[2 \bar{u}_{f}+\right.$ $\varepsilon]=\operatorname{diag}[2 \quad 0.5060]$ and $\tilde{\beta}=\operatorname{diag}\left[1-\left(\bar{u}_{f}-\varepsilon\right)\right]=$ $\operatorname{diag}[1-0.0940]$. Then, based on (4)-(11), the system can be rewritten as

$$
\dot{\tilde{x}}(t)=\left[\begin{array}{cc}
1 & -0.5 \\
1 & 3
\end{array}\right] \tilde{x}(t)
$$

$$
+\left[\begin{array}{cc}
0 & 0.2060 \\
3.00 & 0
\end{array}\right] \operatorname{sat}(v(t))+\left[\begin{array}{c}
0.2060 \\
0
\end{array}\right] \tilde{\omega}(t) \text {. }
$$

According to Theorem 1 with $\eta=1$, the controller for post-fault system $K_{f}$ and the related matrix $P_{f}$ can be computed directly. The center of $\Omega_{x}\left(P_{f}\right)$, which is also the equilibrium point of the post-fault system, is $x_{0}=$ $\left[\begin{array}{ll}-0.4-0.2\end{array}\right]$. Simulation results are shown in Fig. 3 by dashed lines.

Clearly, as shown in Fig. 3(a), the states when the stuck failure is detected and isolated, $x\left(t_{f}\right)$ (marked by the triangle), are inside the stability region of the post-fault system $\Omega_{x}\left(P_{f}\right)$ (the dashed ellipse), $x\left(t_{f}\right) \in$ $\Omega_{x}\left(P_{f}\right)$, which represents the stuck failure, is able to be compensated by the redesigned new controller. This result can also be achieved by $\left[x\left(t_{f}\right)+A^{-1} B \frac{\tilde{\alpha}-\tilde{\beta}}{2} \zeta\right]^{\prime} P_{f}\left[x\left(t_{f}\right)+\right.$ $\left.A^{-1} B \frac{\tilde{\alpha}-\tilde{\beta}}{2} \zeta\right]=0.4338<1$.

Finally, the states are asymptotically convering to the star, which is also the center of the dashed-line ellipse. As shown in Fig. 3(b) the second actuator of the real system is stuck at $\bar{u}_{f}=0.3$. Thus, the number of the available 

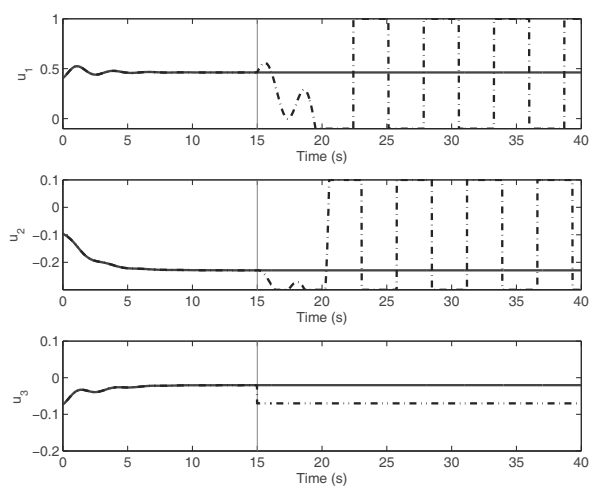

(a)
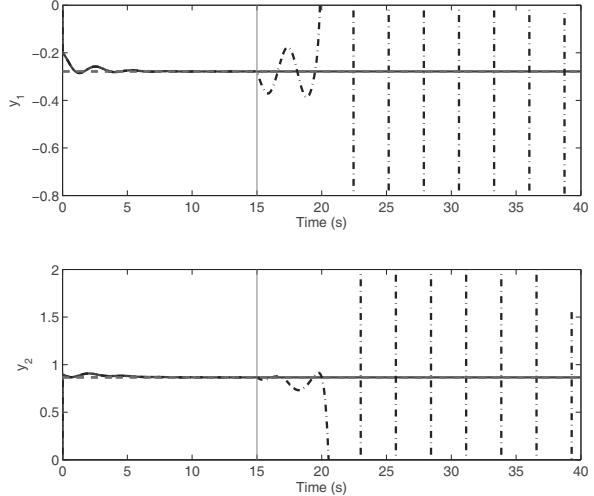

(b)

Fig. 4. Simulation results of a fault-free system and a post-fault system without the proposed control framework for set-point tracking: actuator outputs of the system (28) (a), outputs of the system (28) (b).

control inputs is only one. At the same time, as shown in Fig. 3(c), all control inputs of system (13) are fault-free, which represents the fact that the number of available control inputs is not affected by a stuck failure. Finally, the normalized disturbance produced by (4) is shown in Fig. 3(d)

\subsection{Set-point tracking of a fault-free and a post-fault} system. Consider the following open-loop unstable system:

$$
\begin{aligned}
\dot{x}(t)= & {\left[\begin{array}{ccc}
1.5 & -0.5 & 0.8 \\
1 & 3 & 1.5 \\
0.2 & -1.3 & 0.2
\end{array}\right] x(t) } \\
& +\left[\begin{array}{lll}
1 & 1 & 0 \\
2 & 0 & 3 \\
0 & 1 & 2
\end{array}\right] \operatorname{sat}(u(t)) \\
y(t)= & {\left[\begin{array}{lll}
0 & 1 & 0 \\
0 & 0 & 1
\end{array}\right] x(t) . }
\end{aligned}
$$

The actuator constraints are $\alpha=\operatorname{diag}\left[\begin{array}{lll}1 & 0.1 & 0.1\end{array}\right]$, $\beta=\operatorname{diag}\left[\begin{array}{lll}0.1 & 0.3 & 0.2\end{array}\right]$, and the reference constraints $\rho=1$. For the fault-free condition simulation results are shown in Fig. 4 by solid lines with the initial states $x(0)=\left[\begin{array}{lll}-1.4 & -0.2 & 0.9\end{array}\right]$ and $r=\left[\begin{array}{ll}-0.28 & 0.87\end{array}\right]$. Figure 4(a) shows actuator outputs of the system (28), and Fig. 4(b) shows the outputs of system (28).

Assume that the third actuator is stuck at $\bar{u}_{f}=-0.07$ and detected at $t_{f}=15 \mathrm{~s}$ in the absence of FDI delays. With a fault-free controller, the post-fault system is out of order at last, as shown in Fig. 4 by dot-dashed lines.

In order to guarantee the stability of the post-fault system, the controller should be reconfigured. Taking into account the proposed control framework, the actuator stuck failure is redefined as constraints. Based on Theorem 3 under $\eta=1$, matrix $P_{f}$ and $\varepsilon$ related to controller $\mathcal{K}_{f}$ can be achieved. The new constraints are $\tilde{\alpha}=\operatorname{diag}\left[\begin{array}{lll}1 & 0.1 & -0.0177\end{array}\right]$ and $\tilde{\beta}=\operatorname{diag}\left[\begin{array}{lll}0.1 & 0.3 & 0.1223\end{array}\right]$, with $\varepsilon=0.0523$. Then, the state equation of the system (28) can be rewritten as

$$
\begin{aligned}
\dot{\tilde{x}}(t) & =\left[\begin{array}{ccc}
1.5 & -0.5 & 0.8 \\
1 & 3 & 1.5 \\
0.2 & -1.3 & 0.2
\end{array}\right] \tilde{x}(t)+\left[\begin{array}{c}
0 \\
0.1568 \\
0.1046
\end{array}\right] \tilde{\omega}(t) \\
& +\left[\begin{array}{ccc}
1.6500 & 0.2000 & 0 \\
1.1000 & 0 & 0.1568 \\
0 & 0.2000 & 0.1046
\end{array}\right] \operatorname{sat}(v(t))
\end{aligned}
$$

Figure 5(a) shows the state space of $x(t)$ with stability regions and state trajectories. Note that the closed-loop system has $n+p=5$ dimensions but Fig. 5(a) just shows the state space of $x(t)$ which has 3 dimensions. Obviously, the ellipsoid of $x(t) \in \mathbb{R}^{n}$ is a subset of the ellipsoid of $\xi(t) \in \mathbb{R}^{n+p}$ because of $\xi(t)=\left[\begin{array}{ll}\tilde{x}^{T}(t) e(t)\end{array}\right]^{T}$. Thus, the ellipsoid of $x(t)$ can be graphed according to the following relationship:

$$
\begin{aligned}
\xi^{T}(t) & P_{f} \xi(t) \\
= & {\left[\begin{array}{c}
\tilde{x}(t) \\
e(t)
\end{array}\right]^{T}\left[\begin{array}{ll}
P_{f 11} & P_{f 12} \\
P_{f 12}^{T} & P_{f 22}
\end{array}\right]\left[\begin{array}{c}
\tilde{x}(t) \\
e(t)
\end{array}\right] } \\
= & \left(\tilde{x}(t)-\tilde{x}_{0}(t)\right)^{T} P_{f 11}\left(\tilde{x}(t)-\tilde{x}_{0}(t)\right) \\
& +e^{T}(t)\left[P_{f 22}-P_{f 12}^{T} P_{f 11}^{-1} P_{f 12}\right] e(t) \leq 1,
\end{aligned}
$$

where $P_{f 11} \in \mathbb{R}^{n \times n}, P_{f 22} \in \mathbb{R}^{p \times p}$ and $\tilde{x}_{0}(t)=$ $-P_{f 11}^{-1} P_{f 12} e(t)$. Furthermore, considering $\tilde{x}(t)=x(t)+$ $A^{-1} B \frac{\tilde{\alpha}-\tilde{\beta}}{2} \zeta$, the ellipsoid of $x(t)$ can be achieved according to $P_{f}$. The center and radius are influenced by $e(t)$ and $A^{-1} B \frac{\tilde{\alpha}-\tilde{\beta}}{2} \zeta$. In Fig. 5(a) the stability regions of $x(t)$ at instant $t_{f}$ are shown, where the fault-free system with $P$ is represented by the lower ellipsoid and the post-fault system with $P_{f}$ is represented by the upper 


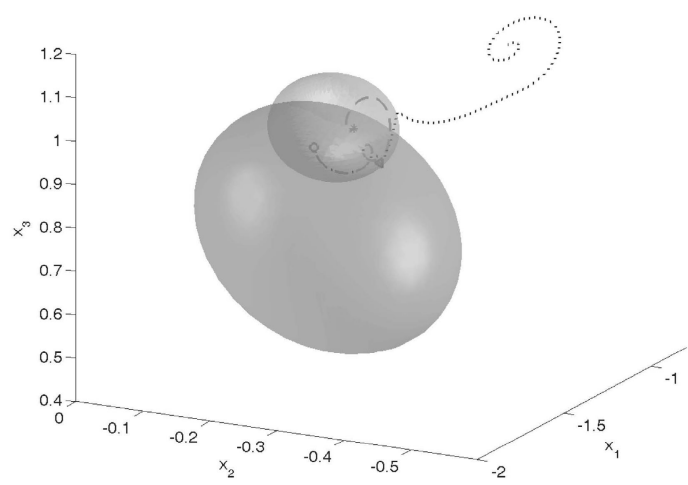

(a)
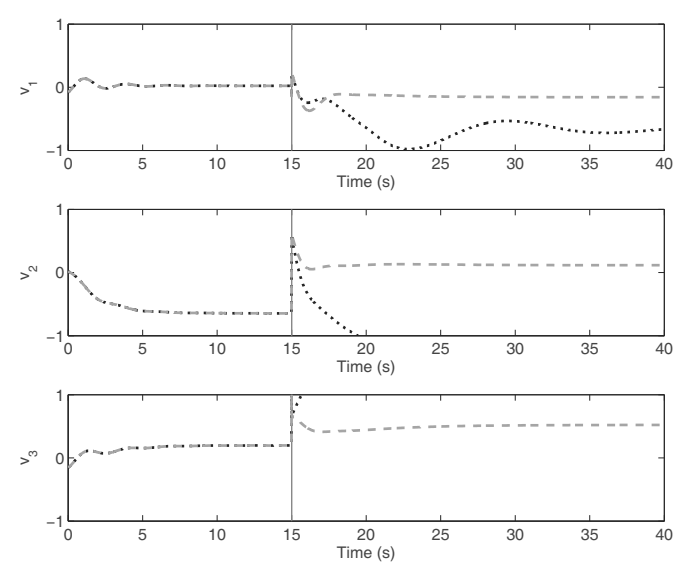

(c)
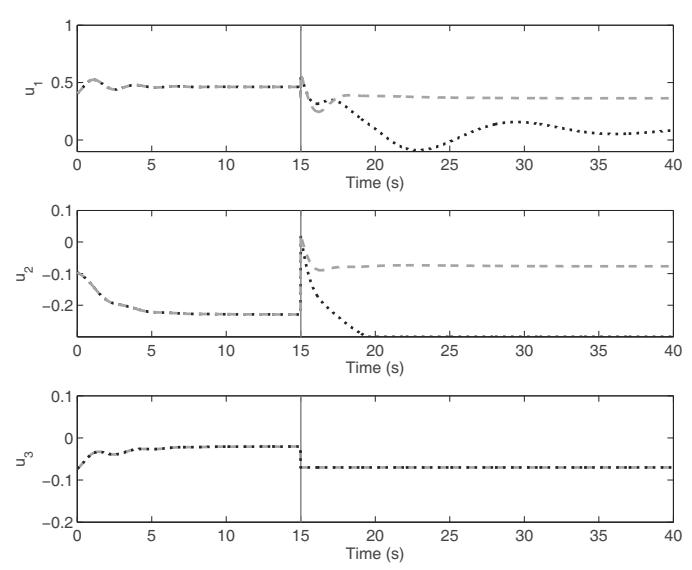

(b)
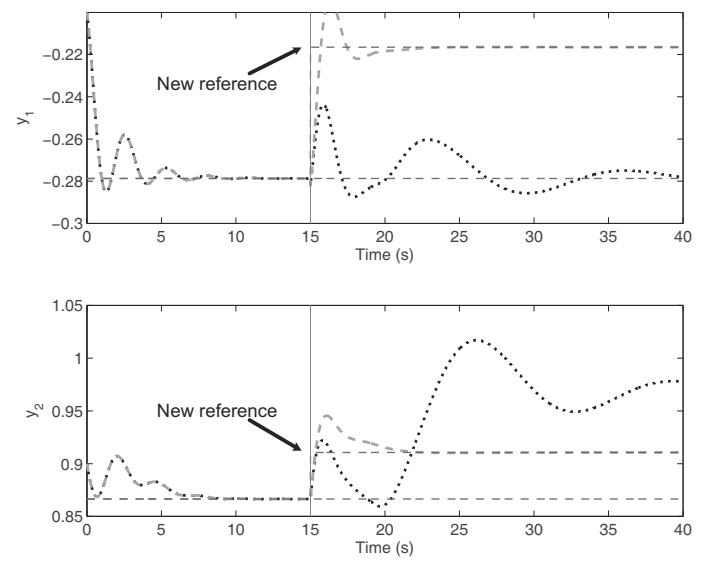

(d)

Fig. 5. Simulation results of a fault-free and a post-fault system for set-point tracking: state space with stability regions and state trajectories (a), actuator outputs of the system (28) (b), control inputs of the system 229) (c), outputs of the system (28) (d).

ellipsoid. The initial states $x(0)$ are marked by the small circle and the states $x\left(t_{f}\right)$ by the triangle. As shown in Fig. 5(a), the states $x\left(t_{f}\right)$ are inside the stability region of the post-fault system $\Omega_{x}\left(P_{f}\right)$, which implies that the stuck failure is able to be compensated by the redesigned new controller.

Figure 5(b) shows actuator outputs of the system (28), where the third actuator is stuck at $\bar{u}_{f}=-0.07$. Figure 5(c) shows the control inputs of the system (16) and, finally, Fig. 5(d) shows the outputs of the system (28). Clearly, the post-fault system with only fault-tolerant controller $\mathcal{K}$ (dot lines) is stable at last, but the post-fault system cannot track the original set-point reference, as shown in Fig. 5(d). In other words, the original reference is unreachable because the second actuator has been saturated, as shown in Fig. 5(b). This conclusion can also be made by $\xi(\infty)^{T} P_{f} \xi(\infty)=$ $24.8452>1$. Thus, reference redesign is required to compute a new reference.

According to Theorem 4, a new reference is calculated as $r=\left[\begin{array}{ll}-0.2165 & 0.9106\end{array}\right]$ and related simulation results are shown in Fig. 5 by dashed lines. As shown in Fig. 5(d), the post-fault system can track the new set-point, and the related control inputs of the controller (17), shown in Fig. 5(b), are not saturated.

\section{Conclusions}

In this paper, actuator stuck failures and actuator constraints were simultaneously considered. For keeping post-fault system safety under actuator constraints, a control framework was developed which involves FTC, reference redesign and system performance evaluation.

Actuator stuck failures were firstly redefined as actuator constraints so that stuck failures and constraints could be unified. A new fault-free system with new constraints was constructed and used to design a 
state-feedback controller to guarantee regional stability of the original post-fault system. Based on the achieved stability region, stuck compensability and reference reachability were analyzed before controller switches to prevent the system from being dangerous. If the original reference were unreachable, the framework would compute a new optimal one instead. Finally, the potential and performance of the proposed method were validated by numerical examples.

In future works, we will focus on improving the universality of the proposed unifying method and research on dynamic reference tracking, etc.

\section{Acknowledgment}

This work was supported by the National Natural Science Foundation of China under the grants U1508208 and U1608253.

\section{References}

Benhayoun, M., Benzaouia, A., Mesquine, F. and Hajjaji, A.E. (2013). System stabilization by unsymmetrical saturated state feedback control, 9th Asian Control Conference (ASCC), Istanbul, Turkey, pp. 1-5.

Cen, Z., Noura, H. and Younes, Y.A. (2015). Systematic fault tolerant control based on adaptive Thau observer estimation for quadrotor UAVs, International Journal of Applied Mathematics and Computer Science 25(1): 159-147, DOI: 10.1515/amcs-2015-0012.

Chen, J. and Patton, R.J. (2012). Robust Model-based Fault Diagnosis for Dynamic Systems, Vol. 3, Springer, Boston, MA.

da Silva, J.M.G. and Tarbouriech, S. (2005). Antiwindup design with guaranteed regions of stability: An LMI-based approach, IEEE Transactions on Automatic Control 50(1): 106-111.

Dardinier-Maron, V., Hamelin, F. and Noura, H. (1999). A fault-tolerant control design against major actuator failures: Application to a three-tank system, Proceedings of the 38th IEEE Conference on Decision and Control, Phoenix, AZ, USA, Vol. 4, pp. 3569-3574.

Famularo, D., Franz, G. and Lucia, W. (2015). Multiple stuck positions actuator faults: A model predictive based reconfigurable control scheme, IEEE Conference on Decision and Control, Osaka, Japan pp. 5091-5096.

$\mathrm{Hu}, \mathrm{T}$., Lin, Z. and Chen, B. M. (2002). An analysis and design method for linear systems subject to actuator saturation and disturbance, Automatica 38(2): 351-359.

Jiang, B. and Chowdhury, F.N. (2005). Fault estimation and accommodation for linear MIMO discrete-time systems, IEEE Transactions on Control Systems Technology 13(3): 493-499.

Jiang, B., Staroswiecki, M. and Cocquempot, V. (2006). Fault accommodation for nonlinear dynamic systems, IEEE Transactions on Automatic Control 51(9): 1578-1583.
Li, Y. and Lin, Z. (2013). Design of saturation-based switching anti-windup gains for the enlargement of the domain of attraction, IEEE Transactions on Automatic Control 58(7): 1810-1816.

Noura, H., Theilliol, D., Ponsart, J. and Chamseddine, A. (2009). Fault-tolerant Control Systems: Design and Practical Applications, Advances in Industrial Control, Springer, Berlin/Heidelberg.

Ossmann, D. and Varga, A. (2015). Detection and identification of loss of efficiency faults of flight actuators, International Journal of Applied Mathematics and Computer Science 25(1): 53-63, DOI: 10.1515/amcs-2015-0004.

Pascal, G. and Pierre, A. (1994). A linear matrix inequality approach to $H_{\infty}$ control, International Journal of Robust and Nonlinear Control 4(4): 421-448.

Qi, X., Qi, J., Theilliol, D., Zhang D., Han, J. and Song, D. (2014). A review on fault diagnosis and fault tolerant control methods for single-rotor aerial vehicles, Journal of Intelligent \& Robotic Systems 73(1-4): 535-555.

Tabatabaeipour, S.M. and Blanke, M. (2014). Calculation of critical fault recovery time for nonlinear systems based on region of attraction analysis, World Congress of the International Federation of Automatic Control, Cape Town, South Africa, pp. 6741-6746.

Theilliol, D., Join, D. and Zhang, Y. (2008). Actuator fault tolerant control design based on a reconfigurable reference input, International Journal of Applied Mathematics and Computer Science 18(4): 553-560, DOI: 10.2478/v10006-008-0048-1.

Wu, F. and Soto, M. (2003). Extended LTI anti-windup control with actuator magnitude and rate saturations, 42nd IEEE Conference on Decision and Control, Maui, HI, USA, Vol. 3, pp. 2786-2791.

Wu, L.B., Yang, G.H. and Ye, D. (2014). Robust adaptive fault-tolerant control for linear systems with actuator failures and mismatched parameter uncertainties, IET Control Theory \& Applications 8(6): 441-449.

Xu, D., Jiang, B. and Shi, P. (2015). Robust NSV fault-tolerant control system design against actuator faults and control surface damage under actuator dynamics, IEEE Transactions on Industrial Electronics 62(9): 5919-5928.

Xu, F., Puig, V., Ocampo-Martinez, C., Olaru, S. and Niculescu, S.-I. (2017). Robust MPC for actuator-fault tolerance using set-based passive fault detection and active fault isolation, International Journal of Applied Mathematics and Computer Science 27(1): 43-61, DOI: 10.1515/amcs-2017-0004.

Yang, G.H., Wang, H. and Xie, L. (2010). Fault detection for output feedback control systems with actuator stuck faults: A steady-state-based approach, International Journal of Robust and Nonlinear Control 20(15): 1739-1757.

Zhang, Y. and Jiang, J. (2003). Fault tolerant control system design with explicit consideration of performance degradation, IEEE Transactions on Aerospace and Electronic Systems 39(3): 838-848. 
Zhang, Y. and Jiang, J. (2008). Bibliographical review on reconfigurable fault-tolerant control systems, Annual Reviews in Control 32(2): 229-252.

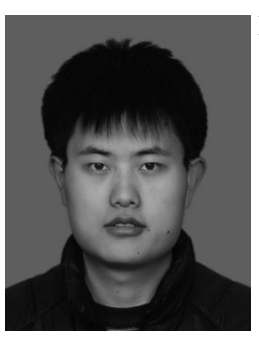

Xin $\mathrm{Qi}$ received the $\mathrm{PhD}$ degree from the Shenyang Institute of Automation, Chinese Academy of Sciences, in 2017. His research interests include fault tolerance control and fault detection for fly-robot with rotor wings

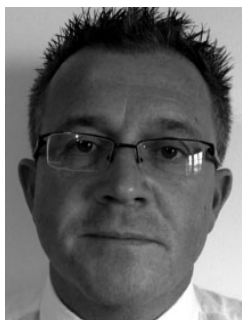

Didier Theilliol received the $\mathrm{PhD}$ degree in control engineering from the University of Lorraine (France) in 1993. Since September 2004, he has been a full professor in the Research Centre for Automatic Control of Nancy (CRAN) at the University of Lorraine, where he coordinates and leads national, European and international R\&D projects in steel industries, wastewater treatment plants and aerospace domains. Didier Theilliol is currently the scientific leader of the research group on co-design of fault tolerant control architectures and methodologies for dynamic systems (CSDF) at CRAN. His research interests include model-based fault diagnosis (FDI) methods, active fault-tolerant control (FTC) system design, and reliability analysis. He has published over $250 \mathrm{journal} /$ conference papers. He is currently an associate editor of various journals such as ISA Transactions. Didier Theilliol was also an associate editor of IEEE Transactions on Reliability (2013-2016).

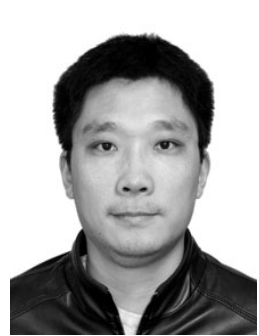

Yuqing He was born in Weihui, China, in 1980 $\mathrm{He}$ received the $\mathrm{BSc}$ degree in engineering and automation from Northeastern University, Qinhuangdao, China, in 2002, and the $\mathrm{PhD}$ degree in pattern recognition and intelligent systems from the Shenyang Institute of Automation, Chinese Academy of Sciences, Shenyang, in 2008. He is currently a professor at the State Key Laboratory of Robotics, Shenyang Institute of Automation. In 2012, he was a visiting researcher at the Institute for Automatic Control Theory, Technical University of Dresden, Germany. His main research interests include nonlinear estimation, control, and cooperation of multiple robots.

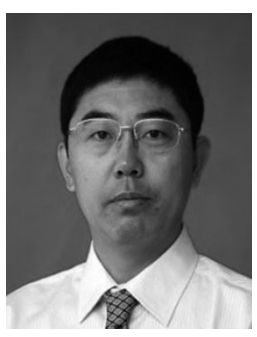

Jianda Han was born in Liaoning, China, in 1968. He received the $\mathrm{PhD}$ degree from the Harbin Institute of Technology, China, in 1998. He is currently a professor and the vice-director of the State Key Laboratory of Robotics, Shenyang Institute of Automation, Chinese Academy of Sciences. His research interests include nonlinear estimation and control, robotics, and mechatronics systems.

Received: 22 November 2016

Revised: 17 May 2017

Re-revised: 10 July 2017

Accepted: 16 July 2017 